\title{
Creencias irracionales y conductas parentales en madres víctimas de violencia infligida por la pareja
}

\author{
Irrational beliefs and parenting behaviors in victim mothers of violence inflicted by the partner
}

Adolfo Aguinaga Álvarez ${ }^{1}$

\section{RESUMEN}

El objetivo del estudio fue establecer las diferencias en las creencias irracionales y las conductas parentales entre un grupo de madres de familia víctimas de violencia infligida por la pareja y un grupo de madres de familia que no se encontraron en esta condición. El propósito fue contribuir a establecer parámetros referenciales sobre la relación madre-hijo en el contexto de la violencia familiar. La investigación fue descriptiva con un diseño comparativo. Se determinó la muestra mediante un muestreo no probabilístico de tipo intencional, y estuvo conformada por dos grupos: Un primer grupo de 100 madres que reportaron ser víctimas de violencia infligida por la pareja y ellas recibieron evaluación psicológica en el Instituto de Medicina Legal de Lima Norte, y un segundo grupo de 92 madres que no fueron víctimas de violencia. La recolección de la información se hizo mediante dos inventarios: El Inventario de Conducta Racional De Shorkey \& Whiteman (1977) y el Inventario de Conducta Parental de Lovejoy (1999). Los estadísticos utilizados fueron la media aritmética, la U de Mann-Whitney, prueba de rangos con signos en pares de Wilcoxon y el coeficiente de correlación de Pearson. Entre los principales resultados se encontró que existen diferencias significativas en las creencias irracionales, siendo las madres maltratadas las que presentaron puntuación más elevada en los factores frustración, necesidad de aprobación, sentimiento de culpa, inercia y evasión, ideas de infortunio y confianza en el control de las emociones. También se encontraron diferencias significativas en las conductas parentales, las madres maltratadas reportaron menor tendencia a presentar conductas hostil/coercitiva y mayor tendencia a presentar conductas de apoyo/compromiso hacia sus hijos. Las implicancias de los hallazgos son discutidas en función a los objetivos, estudios previos y la realidad psicosocial de nuestro país.

PALABRAS CLAVE: Violencia de pareja, creencias irracionales, conductas parentales.

\section{SUMMARY}

The aim of the study was to establish the differences in irrational beliefs and parenting behaviors between a group of family mothers victims of intimate partner violence and a group of family mothers that were not found in this condition. The purpose was to contribute to establishing referential parameters on the mother-child relationship in the context of family violence. The research was descriptive with a comparative design. The sample was determined by a non-probabilistic intentional sampling and consisted on two groups: A first group of 100 mothers who reported being victims of intimate partner violence and they received psychological evaluation at the Institute of Legal Medicine of North Lima, and a second group of 92 mothers who were not victims of violence. The data collection was done through two inventories: The Rational Behavior Inventory from Shorkey \& Whiteman (1977) and The Inventory of Parental Behavior from Lovejoy (1999). The statistics used were the arithmetic mean, the U of MannWhitney, the signed rank test in paires of Wilcoxon and the Pearson correlation coefficient. Among the main results we found that there are significant differences in irrational beliefs, being the battered mothers who had higher scores

Psicólogo Forense. Ministerio Público de Lima Norte. Lima, Perú. 
on the frustration factors, need for approval, feelings of guilt, inertia and avoidance, ideas of misfortune and low confidence in controlling emotions. Significant differences were also found in parental behaviors, battered mothers reported less tendency to hostile / coercive behavior and more likely to have behavior of support / commitment to their children. The implications of the findings are discussed in terms of objectives, previous studies, and the psychosocial reality of our country.

KEYWORDS: Partner violence, irrational beliefs, parental behavior.

\section{INTRODUCCIÓN}

La vulnerabilidad de la mujer frente al fenómeno de la violencia familiar, y más precisamente a la violencia dada en el contexto conyugal, es abordada por autores como Corsi (1992); Sarasua, Zubizarreta, Echeburúa y Corral (2007); Patró, Corbalán y Limiñana (2007); y Domínguez, García y Cuberos (2008); quienes refieren que la mujer que es víctima de maltrato en su relación de pareja puede presentar, generalmente, escasa valoración de sí misma, sentimientos de humillación, vergüenza, miedo, pérdida de control, confusión, sentimiento de culpa, trastorno de estrés post-traumático, ansiedad, depresión, abuso de alcohol y drogas, así como ideación y conducta suicida, entre otras consecuencias negativas.

Estas consecuencias emocionales y conductuales no obedecerían solamente a la situación de maltrato vivida, sino también, a la creencia que se encuentra entre ambas: $\mathrm{A}=$ Situación de maltrato, $\mathrm{B}=$ Creencia sobre dicha situación, $\mathrm{C}=$ Consecuencia emocional y conductual de la creencia sobre la situación de maltrato (Ellis y Grieger, 1981).

En tal sentido, el concepto de "creencia irracional" cobra vital importancia en el contexto de la violencia de pareja. Según Ellis (1962), las creencias irracionales se caracterizan por ser falsas, es decir, no coinciden con la realidad; se expresan como demandas, necesidades o deberes; llevan a emociones inadecuadas como por ejemplo la depresión y la ansiedad y no ayudan a conseguir los objetivos personales. En definitiva, este tipo de creencias propician conductas destructivas para la sociedad y para el individuo (Ellis y Bernard, 1990).

Al respecto, Mc Cann, Skheim y Abrahamson (1988); así como Aguilar, Nightingale (1994); y Barnet (2001), hacen referencia a la afectación del esquema cognitivo de las mujeres víctimas de maltrato; en particular los esquemas de autovalía y autoeficacia, aumentando el riesgo de trastornos como la depresión (Citados por Calvete, Estévez y Corral, 2007).
La violencia en el ámbito conyugal, no solo estaría relacionada con cogniciones o creencias irracionales en las mujeres que se hallan en esta condición, sino también, con las conductas que éstas presentan en el desempeño de sus roles, incluyendo el rol de madre. En tal sentido, la relación de la violencia doméstica y las conductas parentales ha sido abordada por varios autores (Appel y Holden, 1998; Lhor, 2001; Milner, 1993; Nayak y Milner, 1998; Pham, 2000; Zarza y Froján, 2005; Patró, et al, 2007). Ellos encontraron que el estrés asociado a la violencia doméstica es un factor potenciador de prácticas parentales que contemplan el maltrato hacia los hijos. Asimismo, Walker (1994) y Koss (1990) afirman que las víctimas de violencia doméstica emplean violencia con sus hijos hasta ocho veces más que aquellas mujeres separadas o con relaciones no violentas (citados por Zarza y Froján, 2005). Por el contrario, señala Kitzmann (2000) que, una buena relación de pareja se relaciona con el apoyo emocional que recibe la madre, siendo un factor que ayuda en el ejercicio de la parentalidad (citado por Pons - Salvador, Cerezo y Bernabé, 2005).

Sin embargo, existen planteamientos contrarios como los de Levendosky, Lynch y Graham-Bermann (2000), quienes afirman que las mujeres que están involucradas en una relación de violencia doméstica tienen algunos efectos positivos de esta situación en sus conductas parentales, señalando que ellas frecuentemente activan sus recursos personales para responder a la violencia en defensa de sus hijos.

Para un acercamiento teórico más claro hacia el constructo "conducta parental", es importante citar a Lovejoy, et al. (1999), quienes plantearon dos clasificaciones para la conducta parental: la hostilidad/coerción, caracterizada por la expresión de afecto negativo o indiferencia hacia el niño, pudiendo implicar el uso de la coerción, la amenaza o el castigo físico para influenciar la conducta del niño; y el apoyo/ compromiso, reflejado en conductas que demuestran la aceptación del padre hacia el niño por medio del afecto, las actividades compartidas y el apoyo instrumental y emocional (citados por Merino, Díaz \& DeRoma, s.a.). 


\section{Marco teórico}

La violencia conyugal es normalmente precedida por un desacuerdo y posteriormente por un conflicto entre ambos cónyuges, muchos hombres aplican la violencia contra su pareja para resolver dicho conflicto. En esta fase de desacuerdo y de conflicto conyugal, muchos autores hallaron la presencia de creencias irracionales predisponentes a la violencia en este ámbito (Addis \& Bernard, 2002; Epstein, Baucom \& Rankin, 1993; Habke, Hewitt \& Flett, 1999; Kayser \& Himmle, 1994; Metts \& Cupach, 1990); destacándose como una de las causas principales del disturbio en la interacción marital, a las expectativas poco realistas que tienen las parejas de la relación y de su pareja en sí (citados por Hamanci, 2005).

Del mismo modo, Ellis et al. (1989) también identificaron creencias irracionales en los conflictos conyugales que pudieran desencadenar una situación de maltrato (citado por Hamanci, 2005):

a) Exigencia natural (un "debería" dogmático acerca de la conducta del esposo y de la naturaleza del matrimonio).

b) Necesidades (los esposos creen que necesitan ser amados por la pareja, porque de lo contrario serían despreciables).

c) Intolerancia (las parejas se convencen que ellas no pueden resolver los problemas que experimentan o anticipan en sus relaciones).

d) Catastrofizar (las parejas son poco tolerantes cuando no todo es como piensan que deben ser).

e) Condenación (toman los sentimientos de la pareja como un referente del atractivo y del valor humano)

Por su parte, Baucom, Epstein, Sayers y Sher (1989) han descrito cinco categorías de cogniciones que juegan un papel importante en el mal ajuste marital: atención selectiva (implica cada percepción idiosincrática de los eventos que tiene la pareja); atribuciones (la explicación que las parejas dan a los eventos y sucesos); expectativas (predicciones que cada pareja hace acerca de la probabilidad de que determinados eventos ocurran en el futuro); suposición o asunción (las creencias básicas que cada pareja sostiene acerca de la naturaleza de las palabras y las correlaciones entre eventos y características de una relación íntima); normas (creencias de cada pareja acerca de las características de los pensamientos de cada una de ellas o de las relaciones que podría tener) (citados por Hamanci, 2005).
Según Hamanci (2005), muchos estudios han mostrado que hay diferencias de género en las creencias disfuncionales de las relaciones. Por ejemplo, Haferkamp (1994), Stackert y Bursik reportaron que comparadas con los hombres, las mujeres tienen más fuertemente arraigada la creencia que "el desacuerdo o discordia es destructivo". Para los hombres las creencias irracionales más fuertes estaban relacionadas al perfeccionismo sexual en las relaciones de pareja (citados por Hamanci, 2005).

Hamanci (2005), encontró en su estudio que el ajuste marital estaba correlacionado negativamente con las creencias disfuncionales de la relación. Otro hallazgo fue que los sujetos casados que pertenecían al grupo de bajo ajuste tuvieron significativamente más creencias irracionales de la relación que aquellas parejas que poseen un buen ajuste. De acuerdo a este autor, los resultados hallados se apoyan en estudios previos y en las teorías cognitivas conductuales que sostienen la importancia de las creencias irracionales que tienen las parejas en el estrés marital.

Una de las creencias irracionales más frecuente en el marco de la violencia conyugal, es la referida a la creencia de las mujeres de sentirse culpables de ser maltratadas (Sulski; Corsi, 1992; Larraín, 1992; Romero, 2004 y Delgado, 2006). De acuerdo a estos autores, las mujeres maltratadas dentro del rol de esposa y madre, se han ilusionado y han hecho de su familia el proyecto de su vida. Por ello el fracaso del matrimonio es equiparado al fracaso de la vida, y se creen culpables si esto sucede.

Al respecto, Cadillo (2001) y Calvete, et al (2007), coinciden en la identificación de creencias irracionales en el contexto de la violencia de pareja. Las creencias coincidentes en ambos estudios aluden a creencias de culpa, las cuales son identificables en los diversos planteamientos teóricos referidos a las consecuencias en las víctimas de violencia infligida por la pareja.

Cadillo (2001) en una muestra de 233 mujeres peruanas víctimas y no víctimas de violencia conyugal, encontró diferencias significativas en las creencias irracionales de necesidad de aprobación, sentimiento de culpa, inercia y evasión, abatimiento y confianza en el control de las emociones.

Otra de las creencias irracionales destacadas en los estudios realizados sobre la violencia conyugal, es la creencia de la mujer maltratada de depender incondicionalmente del agresor (Matud, Belén y 
Padilla, 2004; Calvete, et al.; Valor - Segura, Expósito y Moya, 2008 y Herman, 1992). Al respecto, señalan que la aplicación sistemática y recurrente del maltrato, afecta el sentido de sí mismo de la víctima en relación con los demás, daña su autonomía y promueve un vínculo patológico con el maltratador. Este proceso va generalmente acompañado del aislamiento a que es sometida la víctima por parte de éste.

Los planteamientos cognitivos conductuales no solo resaltan la importancia de las variables cognitivas, sino también de las variables conductuales. En el marco de la violencia de pareja, las conductas parentales alcanzan relevancia por la trascendencia que destacan algunos autores.

En ese sentido, Troxel y Mathews (2004), Doyle y Markiewicz (2005) refieren que los conflictos en el hogar, reflejados en violencia entre ambos padres, influyen en las conductas que los padres tienen hacia sus hijos, destacando la mayor trascendencia de la madre en la crianza de los hijos y considerándola como el principal factor que estimula las potencialidades del hijo en el ámbito familiar (citado por Musito, Martínez y Murgui, 2006).

Tal como lo señalan los estudios de Gagné, Drapeau, Melancon, Saint-Jacques \& Lepine (2007), así como los de Pons - Salvador, et al (2005), una relación de pareja violenta tiene una notable influencia en la posibilidad de ejercer violencia hacia los hijos. En esa misma línea, la American Bar Association (2005), señala que la relación parental se ve afectada en hogares en los que se da la violencia doméstica, en estas condiciones de violencia, los hijos tienen un cincuenta por ciento de probabilidades de sufrir algún tipo de maltrato por parte de su padre o madre (citado por Sprinkle, 2007).

Por el contrario, Aluja, Barrio \& García (2007) encontraron que las prácticas de crianza definidas por el cariño y aceptación se relacionan con la estabilidad emocional de los padres y con una alta satisfacción de pareja en ellos.

Otros planteamientos sostienen que la madre víctima de violencia de pareja puede asumir una actitud positiva y de apoyo hacia sus hijos, como una forma de protección frente al agresor y su posible agresión contra ellos (Levendosky, et al., 2000).
En la violencia de pareja pueden estar presentes creencias irracionales que han sido identificadas en los estudios anteriormente señalados, así como también, conductas parentales que deriven de esta situación de violencia. Estas creencias y conductas podrían diferir de aquellas que estén presentes en una relación de pareja exenta de violencia, por lo que resulta pertinente dar respuesta a las preguntas que a continuación se plantean.

\section{Formulación del problema}

1) ¿Existen diferencias significativas en las creencias irracionales de un grupo de madres de familia, víctimas de violencia infligida por la pareja, en comparación a un grupo de madres no víctimas de violencia doméstica?

2) ¿Existen diferencias significativas en las conductas parentales de un grupo de madres de familia, víctimas de violencia infligida por la pareja, en comparación en comparación a un grupo de madres no víctimas de violencia doméstica?

\section{Objetivos}

1) Identificar las diferencias de las creencias irracionales de un grupo de madres de familia víctimas de violencia inflingida por la pareja, en comparación a un grupo de madres de familia no víctimas de violencia doméstica.

2) Identificar las diferencias de las conductas parentales de un grupo de madres de familia víctimas de violencia inflingida por la pareja, en comparación a un grupo de madres de familia no víctimas de violencia doméstica.

\section{MÉTODO}

Muestra: La muestra estuvo conformada por dos grupo de 100 madres de familia, entre 20 y 40 años que asistieron a evaluación psicológica al Instituto de Medicina Legal del Ministerio Público, sede Lima Norte, y que reportaron ser víctimas de violencia infligida por la pareja; y por un grupo de 92 madres que no reportaron encontrarse en esa condición.

La investigación fue de tipo transversal y diseño descriptivo comparativo; siendo las variables de estudio las creencias irracionales y las conductas parentales. 
CUADRO 1: Descripción y distribución de ítems por factor del Inventario de Conducta Racional de Shorkey \& Whiteman (Shorkey \& Whiteman, 1977). (Citados por Díaz, 2002).

\begin{tabular}{|c|c|c|}
\hline FACTORES & DESCRIPCIÓN & ÍTEM \\
\hline I. Frustración & $\begin{array}{l}\text { Capacidad para responder de manera racional ante las situaciones difíciles y } \\
\text { frustraciones, logrando reconocer de modo objetivo la situación y sus consecuencias. }\end{array}$ & $\begin{array}{l}06,11 \\
25,30\end{array}$ \\
\hline II. Exceso de culpa & $\begin{array}{l}\text { Auto atribución o Hetero atribución de culpa, por desviaciones de los valores } \\
\text { tradicionales. }\end{array}$ & $\begin{array}{l}03,08, \\
32\end{array}$ \\
\hline III. Perfeccionismo & Demanda de perfección en todas las áreas de la conducta. & $\begin{array}{l}12,14 \\
16,28\end{array}$ \\
\hline $\begin{array}{l}\text { IV. Necesidad de } \\
\text { aprobación }\end{array}$ & Demanda de que sus atributos, ideas y conductas sean aprobadas por los demás. & $\begin{array}{l}17,18, \\
19\end{array}$ \\
\hline $\begin{array}{l}\text { V. Necesidad de ayuda y } \\
\text { cuidados }\end{array}$ & Demanda de que la gente cuide y ayude a otras personas. & $\begin{array}{l}01,02 \\
09,13\end{array}$ \\
\hline VI. Sentimiento de culpa & Culpa y pena del yo y otros por errores, pecados e injusticias. & $\begin{array}{l}27,29, \\
31,33\end{array}$ \\
\hline VII. Inercia y evasión & $\begin{array}{l}\text { Tendencia a evitar situaciones difíciles o de cierta tensión con aceptación de dificultad } \\
\text { y grado en el cual las personas desean enfrentarse a situaciones desagradables pero } \\
\text { necesarias. }\end{array}$ & $\begin{array}{l}05,10, \\
21\end{array}$ \\
\hline $\begin{array}{l}\text { VIII. Grado de } \\
\text { independencia }\end{array}$ & $\begin{array}{l}\text { Aceptación de independencia en las decisiones y también en las consecuencias de las } \\
\text { acciones. }\end{array}$ & $\begin{array}{l}24,36, \\
37\end{array}$ \\
\hline IX. Aprobación & Tendencia a trastornarse por la evaluación negativa de los demás. & $\begin{array}{l}15,20, \\
31\end{array}$ \\
\hline $\mathrm{X}$. Ideas de infortunio & $\begin{array}{l}\text { Tendencia a trastornarse basándose en el pensamiento de posible desgracia y a la } \\
\text { probabilidad que ocurra. }\end{array}$ & $\begin{array}{l}23,26, \\
35\end{array}$ \\
\hline $\begin{array}{l}\text { XI. Confianza en el control } \\
\text { de emociones }\end{array}$ & Capacidad para ejercer dominio sobre las emociones. & $\begin{array}{l}04,07 \\
22,34\end{array}$ \\
\hline
\end{tabular}

CUADRO 2: Descripción y distribución de ítems por cada valor del Inventario de Conducta Parental de Lovejoy, M.C., Weis, R., O'Hara, E. \& Rubin, E.R.

\begin{tabular}{|c|c|c|}
\hline VALORES & DESCRIPCIÓN & ÍTEM \\
\hline Hostil/Coercitiva & $\begin{array}{l}\text { Conducta que expresa afecto negativo o indiferencia hacia el niño, pudiendo } \\
\text { implicar el uso de la coerción, la amenaza o el castigo físico para influenciar } \\
\text { en la conducta del niño. }\end{array}$ & $\begin{array}{l}01,03,05,07,09 \\
13,15,17,19,20\end{array}$ \\
\hline Apoyo/Compromiso & $\begin{array}{l}\text { Conducta que demuestra la aceptación del padre hacia el niño por medio } \\
\text { del afecto, las actividades compartidas y el apoyo instrumental, emocional. }\end{array}$ & $\begin{array}{l}02,14,06,08, \quad 10 \\
11,12,14,16,18\end{array}$ \\
\hline
\end{tabular}

\section{Instrumentos de Recolección de la Información}

Inventario de Conducta Racional (Shorkey \& Whiteman, 1977).- El Inventario de Conducta Racional fue elaborado por Shorkey y Whiteman (1997), consta de 37 ítems, divididos en 11 factores. El instrumento cuenta con una confiabilidad aceptable obtenida por el alfa de Cronbach $\mathrm{r}=.83$ en ambos grupos de comparación.

Inventario de Conducta Parental (ICP) (Lovejoy, M.C., Weis, R., O'Hara, E. \& Rubin, E.R.).El diseño de su construcción siguió un procedimiento reportado en Lovejoy et al. (1999), que apuntaba hacia la evaluación de amplio rango de la conducta parental en dos dominios: la conducta hostil/coercitiva y de apoyo/compromiso. El inventario contiene 20 afirmaciones de conductas específicas, en que la madre debe responder sobre el grado de certeza con el que realiza cada una de ellas en la relación con su hijo. Las madres responden sobre una escala del cero (nada cierto) hasta el 5 (muy cierto) y en alguno de los tres marcos de tiempo: conducta del presente día, durante la semana y generalmente.

En la validación de contenido, expertos en desarrollo infantil comunicaron la relevancia y representatividad de los ítems, que en una escala del 1 al 5 tuvieron índices promedio de 4,28 y 4,51 para hostilidad/ coerción y soporte/compromiso, respectivamente. 
La validez de constructo se evaluó con sucesivas aplicaciones del análisis factorial confirmatorio, obteniendo la estructura bidimensional hipotetizada.

El alfa de Cronbach reportado para este instrumento fue de .76 para ambos grupos de comparación.

Los estadísticos utilizados fueron la U de MannWhitney y el Coeficiente de correlación de Pearson. El nivel de significancia fue de $.01 \mathrm{y}$ de .05 .

\section{RESULTADOS}

A continuación se presentarán los resultados en función a las hipótesis formuladas:
Al analizar el rango promedio de la creencia irracional sentimiento de culpa en la Tabla 1, se encontró una diferencia a favor de las madres que reportaron maltrato (113.57), mientras que las madres que no se hallaron en dicha situación presentaron una cifra inferior (77.94). Al complementar este hallazgo con las diferencias significativas $(\mathrm{p}<.01)$ obtenidas en el análisis de la U de Mann-Whitney (ver Tabla 2), se puede inferir que las madres maltratadas por sus parejas serían más proclives a sentir culpa y pena de sí mismas y de los demás por errores, pecados e injusticias.

La creencia irracional inercia y evasión alude a la tendencia a evitar situaciones difíciles, o de cierta

TABLA 1: Promedio de rangos de las creencias irracionales según los grupos de comparación

\begin{tabular}{|c|c|c|c|c|}
\hline Creencias irracionales & Maltrato & $\mathrm{N}$ & Rango promedio & Suma de rangos \\
\hline Frustación & $\begin{array}{l}\text { Con maltrato } \\
\text { Sin maltrato } \\
\text { Total }\end{array}$ & $\begin{array}{r}100 \\
92 \\
192\end{array}$ & $\begin{array}{r}106.28 \\
85.87\end{array}$ & $\begin{array}{r}10628.00 \\
7900.00\end{array}$ \\
\hline Exceso de culpa & $\begin{array}{l}\text { Con maltrato } \\
\text { Sin maltrato } \\
\text { Total }\end{array}$ & $\begin{array}{r}100 \\
92 \\
192\end{array}$ & $\begin{array}{r}103.14 \\
89.28\end{array}$ & $\begin{array}{r}10314.00 \\
8214.00\end{array}$ \\
\hline Perfeccionismo & $\begin{array}{l}\text { Con maltrato } \\
\text { Sin maltrato } \\
\text { Total }\end{array}$ & $\begin{array}{r}100 \\
92 \\
192\end{array}$ & $\begin{array}{l}97.39 \\
95.53\end{array}$ & $\begin{array}{l}9739.00 \\
8789.00\end{array}$ \\
\hline Necesidad de aprobación & $\begin{array}{l}\text { Con maltrato } \\
\text { Sin maltrato } \\
\text { Total }\end{array}$ & $\begin{array}{r}100 \\
92 \\
192\end{array}$ & $\begin{array}{l}113.06 \\
78.51\end{array}$ & $\begin{array}{r}11305.50 \\
7222.50\end{array}$ \\
\hline Necesidad de ayuda y cuidados & $\begin{array}{l}\text { Con maltrato } \\
\text { Sin maltrato } \\
\text { Total }\end{array}$ & $\begin{array}{r}100 \\
92 \\
192\end{array}$ & $\begin{array}{l}99.86 \\
92.85\end{array}$ & $\begin{array}{l}9986.00 \\
8542.00\end{array}$ \\
\hline Sentimiento de culpa & $\begin{array}{l}\text { Con maltrato } \\
\text { Sin maltrato } \\
\text { Total }\end{array}$ & $\begin{array}{r}100 \\
92 \\
192\end{array}$ & $\begin{array}{r}113.57 \\
77.94\end{array}$ & $\begin{array}{r}11357.50 \\
7170.50\end{array}$ \\
\hline Inercia y evasión & $\begin{array}{l}\text { Con maltrato } \\
\text { Sin maltrato } \\
\text { Total }\end{array}$ & $\begin{array}{r}100 \\
92 \\
192\end{array}$ & $\begin{array}{r}110.04 \\
81.78\end{array}$ & $\begin{array}{r}11004.50 \\
7523.50\end{array}$ \\
\hline Grado de independencia & $\begin{array}{l}\text { Con maltrato } \\
\text { Sin maltrato } \\
\text { Total }\end{array}$ & $\begin{array}{r}100 \\
92 \\
192\end{array}$ & $\begin{array}{l}98.01 \\
94.85\end{array}$ & $\begin{array}{l}9801.50 \\
8726.50\end{array}$ \\
\hline Aprobación & $\begin{array}{l}\text { Con maltrato } \\
\text { Sin maltrato } \\
\text { Total }\end{array}$ & $\begin{array}{r}100 \\
92 \\
192\end{array}$ & $\begin{array}{r}115.16 \\
76.22\end{array}$ & $\begin{array}{r}11516.00 \\
7012.00\end{array}$ \\
\hline Ideas de infortunio & $\begin{array}{l}\text { Con maltrato } \\
\text { Sin maltrato } \\
\text { Total }\end{array}$ & $\begin{array}{r}100 \\
92 \\
192\end{array}$ & $\begin{array}{r}109.96 \\
81.88\end{array}$ & $\begin{array}{r}10995.50 \\
7532.50\end{array}$ \\
\hline Confianza en el control de las emociones & $\begin{array}{l}\text { Con maltrato } \\
\text { Sin maltrato } \\
\text { Total }\end{array}$ & $\begin{array}{r}100 \\
92 \\
192\end{array}$ & $\begin{array}{c}105.27 \\
86.97\end{array}$ & $\begin{array}{r}10527.00 \\
8001.00\end{array}$ \\
\hline
\end{tabular}


tensión con aceptación de dificultad y grado, en este caso las personas desean enfrentarse a situaciones desagradables pero necesarias. En tal sentido, los resultados de la Tabla 1 indican que las madres maltratadas presentan una mayor tendencia hacia esta creencia irracional que las madres no maltratadas, siendo el rango promedio de 110.04 para el primer grupo y de 81.78 para el segundo grupo de madres. Las diferencias son significativas entre ambos grupos $(\mathrm{p}<.01)$ según la $\mathrm{U}$ de Mann Whitney (ver Tabla 2).

Por otro lado, se observan diferencias en el rango promedio de la creencia irracional aprobación (ver Tabla 1); diferencias a favor del grupo de madres que reportan maltrato de sus parejas (115.16) respecto al otro grupo de comparación (76.22). Según el Análisis mediante la U de Mann-Whitney en la Tabla 2, estas diferencias serían significativas $(\mathrm{p}<.01)$, lo que refleja que las madres maltratadas tienden más a trastornarse por la critica negativa de los demás, que las madres que no son maltratadas.

Según los resultados de la Tabla 1, se puede apreciar que en la creencia irracional ideas de infortunio, el grupo de madres maltratadas presenta un promedio de $109.96 \mathrm{y}$ el de madres sin maltrato 81.88, observándose una marcada diferencia a favor del primer grupo. Esta diferencia es significativa mediante la U de Mann-Whitney con un nivel de .01 (ver Tabla 2). Esto indica que las madres víctimas de maltrato tienden a trastornarse, en mayor medida, que las madres no maltratadas, porque creen en una posible desgracia y en la probabilidad que esta ocurra.

Finalmente, los resultados de la Tabla 1 muestran diferencias de rango promedio en la creencia irracional confianza en el control de las emociones, siendo 105.27 para el grupo de madres maltratadas y 86.97 para el grupo de madres que no lo son. Según el análisis con la $\mathrm{U}$ de Mann-Whitney estas diferencias son significativas ( $\mathrm{p}<.05)$ (ver Tabla 2), lo que indicaría que las madres maltratadas tienen menos capacidad para controlar sus emociones que aquellas madres que no son víctimas de maltrato.

\section{Análisis de las diferencias de las conductas parentales en los grupos de comparación}

En las siguientes tablas se analizan los rangos promedio de puntuación obtenidos por ambos grupos según cada valor del Inventario de Conducta Parental, así como las diferencias estadísticas entre los grupos comparados mediante la U de Mann-Whitney. Las tablas serán descritas y analizadas conjuntamente.

En la Tabla 3 se puede apreciar que la conducta parental hostil/coercitiva en las madres maltratadas, presentaron un rango promedio de 86.55, que es menor al obtenido por las madres que no refieren maltrato, 107.32. Esta diferencia es significativa según el análisis de la Tabla $4(\mathrm{p}<.05)$, lo que indica que las madres maltratadas tienden a expresar afecto negativo

TABLA 2: Diferencia estadística en las creencias irracionales según los grupos de comparación

\begin{tabular}{lrr}
\hline Creencias irracionales & $\begin{array}{c}\text { U de } \\
\text { Mann-Whitney }\end{array}$ & $\begin{array}{r}\text { Sig. asintót. } \\
\text { (bilateral) }\end{array}$ \\
\hline Frustración & 3622.000 & $.010^{*}$ \\
Exceso de culpa & 3936.000 & .081 \\
Perfeccionismo & 4511.000 & .815 \\
Necesidad de aprobación & 2944.500 & $.000^{* *}$ \\
Necesidad de ayuda y cuidados & 4264.000 & .377 \\
Sentimiento de culpa & 2892.500 & $.000^{* *}$ \\
Inercia y evasión & 3245.500 & $.000^{* *}$ \\
Grado de independencia & 4448.500 & .690 \\
Aprobación & 2734.000 & $.000^{* *}$ \\
Ideas de infortunio & 3254.500 & $.000^{* *}$ \\
Confianza en el control de las emocion & 3723.000 & $.022^{*}$ \\
\hline
\end{tabular}

*. Diferencia significativa $\mathrm{p}<.01$

**. Diferencia significativa $\mathrm{p}<.05$ 
TABLA 3: Promedio de rangos de las conductas parentales según los grupos de comparación

\begin{tabular}{rlrcc}
\hline Conductas parentales & Maltrato & N & Rango promedio & Suma de rangos \\
\hline \multirow{3}{*}{ Hostil/Coercitiva } & Con maltrato & 100 & 86.55 & 8655.00 \\
& Sin maltrato & 92 & 107.32 & 9873.00 \\
& Total & 192 & & \\
Apoyo/Compromiso & Con maltrato & 100 & 107.63 & 10763.00 \\
& Sin maltrato & 92 & 84.40 & 7765.00 \\
& Total & 192 & & \\
\hline
\end{tabular}

TABLA 4: Diferencia estadística en las conductas parentales según los grupos de comparación

\begin{tabular}{lcc}
\hline & \multicolumn{2}{c}{ Estadístico } \\
\cline { 2 - 3 } Conductas & $\begin{array}{c}\text { Ude } \\
\text { parentales }\end{array}$ & $\begin{array}{c}\text { Sig.asintót. } \\
\text { (bilateral) }\end{array}$ \\
\hline Hostil/Coercitiva & 3605.000 & $.010^{*}$ \\
Apoyo/Compromiso & 3487.00 & $.004^{* *}$ \\
\hline ** Diferencia significativa $\mathrm{p}<.01$ & \\
$*$ Diferencia significativa $\mathrm{p}<.05$ &
\end{tabular}

(amenazas, castigos, indiferencia, entre otros) hacia sus hijos, en menor medida que aquellas madres que no son maltratadas.

Por el contrario, al analizar el rango promedio de la conducta parental apoyo/compromiso en la Tabla 3, se observa que las madres maltratadas obtienen una puntuación mayor que las madres no maltratadas, siendo el rango promedio para las primeras 107.63 y para las últimas 84.40. Mediante la U de MannWhitney se aprecia en la Tabla 4, que estas diferencias son estadísticamente significativas $(\mathrm{p}<.01)$. Ello indicaría que las madres que son víctimas de maltrato por parte de sus parejas presentan una mayor tendencia a expresar conductas de aceptación, afecto, y apoyo emocional hacia sus hijos, que las madres que no son maltratadas.

\section{DISCUSIÓN}

El análisis de los resultados indica diferencias significativas en siete creencias irracionales de las once que han sido estudiadas, siendo ello compatible con con el objetivo de investigación: Identificar las diferencias en las creencias irracionales de un grupo de madres de familia víctimas de violencia infligida por la pareja en comparación a un grupo de madres no víctimas de violencia infligida por la pareja. A continuación se analizarán estas diferencias encontradas.
De acuerdo a lo hallado, las madres que sufren maltrato tendrían más dificultades para responder de manera racional ante situaciones frustrantes y para reconocer de modo objetivo la situación y sus consecuencias, que aquellas madres que no serían víctimas de maltrato. Este hallazgo no concuerda con el trabajo de Cadillo (2001) quien no encontró diferencias en la creencia irracional de "frustración" en mujeres víctimas y no víctimas de violencia doméstica.

Cabe resaltar, que la presente investigación considera la condición de madre de familia como criterio de inclusión para la muestra, mientras que el estudio de Cadillo (2001) no lo hizo. De acuerdo a ello, se puede inferir que las mujeres que son madres y que son maltratadas por sus parejas tendrían más posibilidades de tolerar menos la frustración que aquellas mujeres que también son maltratadas, pero no son madres de familia.

Posiblemente, el tener hijos es una condición que influye en la capacidad de aceptar las situaciones adversas o frustraciones de las mujeres que son víctimas de maltrato. Este hallazgo sugiere dos posibles elementos estresantes en la muestra estudiada, y que pueden estar vinculadas a una mayor frustración: El rol de madre y la situación de maltrato que atraviesan. 
Otra diferencia en las creencias irracionales de ambos grupos de estudio, se centra en la mayor necesidad de aprobación y en la mayor tendencia a sentirse trastornadas por la critica negativa de los demás que presentan las madres que reportan maltrato de sus parejas. Este resultado puede relacionarse con lo encontrado por Calvete y Cardeñoso (1999), quienes hallaron asociación entre la creencia de necesidad de aprobación y los síntomas depresivos. Asimismo, los resultados coinciden con el trabajo de Cadillo (2001), quien encontró diferencias en la necesidad de aprobación entre mujeres que son víctimas de maltrato $\mathrm{y}$ aquellas que no lo son.

La necesidad de aprobación de una mujer que es víctima de maltrato podría estar basada en una baja autoestima. Según Larraín (1992); así como, Orava, Mc Leod y Sharpe (1996) (citados por Matud, et al, 2004), cuando la mujer es agredida por su pareja, de forma repetida, puede disminuir su autoestima y su sentimiento de autoeficacia, por lo que necesitaría un significativo refuerzo externo (aprobación) para recuperar su sentido de valía.

Otra creencia que predomina en las madres que padecen de maltrato es el sentimiento de culpa, lo muestran en mayor proporción que las madres que no reportan maltrato. Este hallazgo sugiere que la situación de maltrato estaría motivando a las madres a sentir culpa y pena de sí mismas y de los demás por errores, pecados e injusticias.

Lo anterior es consistente con las investigaciones de Cadillo (2001) y Calvete, et al., concordando además, con los planteamientos teóricos de Sulszki (s.f.) y Delgado (2006), quienes refieren que las mujeres víctimas de maltrato llegan a creer que tienen la culpa de ser maltratadas. Su situación de maltrato puede generar en ellas un estado de confusión que las lleva a percibirse culpables de las agresiones recibidas (Corsi, 1992).

El sentimiento de culpa podría estar sustentado, además, en la valoración extrema que la mujer atribuye a la relación de pareja, concibiéndola como un proyecto de vida que la define como persona y como mujer. Al ver fracasada su relación conyugal y ver que sus expectativas de consolidación personal se frustran con ello, tienden a creer que han fallado como mujer y sentirse culpables de ello (Romero, 2004 y Delgado, 2006).
Otra de las diferencias encontradas en las creencias irracionales de ambos grupos de madres, alude a una mayor presencia de la creencia inercia y evasión en las madres con maltrato. Es decir, que las madres que serían agredidas por sus parejas tenderían a evitar situaciones difíciles o de cierta tensión.

Esto podría deberse a que la mujer que es víctima de maltrato suele percibirse con pocos recursos personales de afronte ante las demandas o riesgos de su entorno, optando por una postura pasiva e inerte (Herman, 1992; Larraín, 1992; Matud, 2004; Romero, 2004; Orava, Mc Leod y Sharpe, 1996). Al respecto, Albertín (2006), señala que en la mujer maltratada surge una creencia de "incapacidad" frente a simples eventos cotidianos que suceden en su entorno, unido a sentimientos de fracaso personal por no ser capaz de afrontar la vida cotidiana.

En el análisis de la creencia irracional ideas de infortunio, se ha encontrado en el grupo de madres que reportan maltrato, una mayor tendencia a trastornarse basándose en la creencia de una posible desgracia y a la probabilidad que ocurra. Este resultado contradice lo encontrado por Cadillo (2001), quien no halló diferencias en esta creencia irracional entre mujeres maltratadas y no maltratadas por sus parejas.

El hallazgo descrito sugiere una mayor presencia de ansiedad anticipatoria en las madres víctimas de violencia de pareja, que las pueden conducir a evaluar como riesgosas situaciones que no necesariamente lo son. Los indicadores de ansiedad en las mujeres maltratadas representan una característica común en ellas, según referencia de numerosos autores (Matud, et al.). Esta ansiedad podría explayarse más allá de la relación violenta en la que estaría inmersa y focalizarse también hacia estímulos externos a ella.

En tal sentido, Flores y Velasco (2003) encontraron que una de las creencias irracionales más frecuentes en las mujeres víctimas de violencia conyugal fue "se debe sentir miedo o ansiedad ante cualquier cosa desconocida, incierta o potencialmente peligrosa". Otra creencia encontrada en este estudio fue "los acontecimientos externos son la mayoría de las desgracias de la humanidad", siendo ambas creencias compatibles con lo explicado en el párrafo anterior.

Por otro lado, los resultados indican que las madres que refieren violencia de sus parejas tendrían menos capacidad de control sobre sus emociones que aquellas madres que no son víctimas de maltrato. 
Este hallazgo concuerda con lo planteado por autores como Corsi (1992); Sarasua, et al. (2007); Patrón, et al.; y Dominguez, García y Cuberos (2008), los cuales refieren que un efecto de la violencia sobre la mujer es la pérdida de control. Asimismo, se observa coincidencia con el estudio de Cadillo (2001), el cual señala diferencias significativas en la creencia irracional de confianza en el control de las emociones entre mujeres que son víctimas de violencia doméstica y aquellas que no lo son.

La percepción de incontrolabilidad que tienen estas mujeres puede deberse a los continuos fracasos que tienen al buscar salir de una relación de violencia, perdiendo cada vez más la confianza en sí misma y en su capacidad de asumir el control de su vida. Ello le produce a las mujeres maltratadas sentimientos de enojo y enfado contra sí y contra los que le rodean por haber tenido, anteriormente al suceso, la ilusión de control (Albertín, 2006).

El otro objetivo de la investigación, alude a las diferencias significativas en las conductas parentales encontradas en ambos grupos de estudio. Sin embargo, estas diferencias son contrarias a lo que indica la mayor parte de planteamientos teóricos y empíricos; es decir, las madres que son víctimas de maltrato por parte de sus parejas, tenderían también a tener una conducta parental negativa, orientada hacia la agresión y punición (Edleson, 1999 y Appel y Holden, 1998) (citados por Patró y Limiñana, 2005).

En las de madres maltratadas se observa una menor tendencia a mostrarse hostiles con sus hijos que aquellas madres que no son víctimas de maltrato por sus parejas. Por el contrario, las madres que reportan maltrato, describen una mayor posibilidad de presentar conductas de apoyo, compromiso y afecto hacia sus hijos, que el grupo de madres no maltratadas.

De acuerdo a lo anterior, se podría resaltar que las madres víctimas de maltrato, presentan una mayor tendencia a expresar conductas de aceptación, afecto, y apoyo emocional hacia sus hijos, que las madres que no son maltratadas.

Una posible explicación de lo dicho antes, estaría relacionada con los planteamientos de Levendosky, et al.(2000), quienes afirmaron que algunas mujeres que padecen el estrés de una relación de pareja conflictiva y violenta, pueden activar sus recursos personales para afrontar esta violencia y proteger a sus hijos ante el agresor. Asimismo, se produciría una "identificación negativa" con el agresor en el proceso de maltrato.
Este concepto alude a que la persona adopta actitudes y conductas opuestas a las que el agresor exhibe (Roca, 2002). En este caso, la muestra de madres que manifestaron sufrir maltrato, adoptarían una conducta contraria a la violencia que padecen, siendo más bien, afectuosas y comprometidas con sus hijos.

\section{CONCLUSIONES}

1. Existen diferencias significativas en las creencias irracionales entre las madres que reportan violencia de su pareja y aquellas que no reportan maltrato por su pareja.

2. Las puntuaciones en las creencias irracionales frustración, necesidad de aprobación, sentimiento de culpa, inercia y evasión, aprobación, ideas de infortunio y confianza en el control de las emociones, son más altas en las madres que son maltratadas por su pareja respecto de aquellas que no lo son.

3. Existen diferencias significativas en las conductas parentales entre las madres que reportan ser víctimas de violencia por parte de su pareja y aquellas que no se encuentran en dicha condición.

4. Las madres víctimas de maltrato por su pareja evidencian una mayor conducta parental apoyo/ compromiso, mientras que las madres que no son víctimas de violencia tienden más a presentar una conducta parental hostil/coercitiva.

\section{REFERENCIAS BIBLIOGRÁFICAS}

1. Albertín, P. (2006). Psicología de la victimización criminal. En: M. Soria \& D. Sáiz. (Coord.). Psicología Criminal. Madrid: Pearson Educación S.A.

2. Aluja, A., Barrio, V. \& García, L. (2007). Personality, social values, and marital satisfaction as predictors of parents' rearing styles. International Journal of Clinical and Health Psychology; 7(3), 725-737.

3. Appel, A.E. \& Holden, G.W. (1998). The cooccurrence of spouse and physical child abuse: A review and appraisal. Journal of Family Psychology, $12,578-599$.

4. Cadillo, M. (2001). Creencias irracionales en mujeres víctimas y no víctimas de la violencia conyugal. Tesis para licenciado en Psicología. Universidad Nacional Federico Villarreal, Lima, Perú.

5. Calvete, E. \& Cardeñoso, O. (1999). Creencias y síntomas depresivos: Resultados preliminares en el desarrollo de una Escala de Creencias Irracionales abreviada. Anales de psicología, 15(2), 179-190

6. Calvete, E., Estévez, A. \& Corral, S. (2007). Trastorno por estrés postraumático y su relación con esquemas cognitivos disfuncionales en mujeres maltratadas. Psicothema, 19(3), 446-451. 
7. Corsi, J. (1992). Algunas cuestiones básicas sobre la violencia familiar. Buenos Aires: Universidad Autónoma de Buenos Aires.

8. Delgado, C. (2006). Patrones de masculinidad y feminidad asociados al ciclo de la violencia de género. Salamanca: Universidad Pontificia de Salamanca.

9. Domínguez, J., García, P. \& Cuberos, I. (2008). Violencia contra las mujeres en el ámbito doméstico: consecuencias sobre la salud psicosocial. Anales de Psicología; 24, 1, 115-120.

10. Ellis, A. (1962). Reason and emotion in Psychoterapy. New York: Springer.

11. Ellis, A. \& Grieger, R. (1981). Manual de Terapia Racional Emotiva. Bilbao: Editorial Desclee de Brouwer, S.A.

12. Ellis, A. \& Bernard, M. (1990). ¿Qué es la Terapia Racional Emotiva (RET)?. En: A. Ellis \& R. Grieger (editor), Manual de Terapia Racional emotiva. Volumen 2. Bilbao: Desclée de Brouwer.

13. Flores, A. \& Velasco, C. (2003). Miedos y creencias irracionales en mujeres víctimas de violencia familiar. Ajayu,2(1).

14. (Citado en Marzo del 2009) Disponible en: http:// www.ucb.edu.bo/publicaciones/ajayu/v2n1/v2n1a4. html

15. Gagné, M., Drapeau, S., Melancon, C., Saint-Jacques, M. \& Lepine, R. (2007). Links Between Parental Psychological Violence, Other Family Disturbances, and Children's Adjustment. Family Process, 4(46), 523-542.

16. Hamanci, Z. (2005). Dysfunctional relationship beliefs in marital satisfaction and adjustment. Social Behavior and Personality; 33(4), 313-328.

17. Herman, J. (1992). Complex PTSD: A syndrome in survivors of prolonged and repeat trauma. Journal of Traumatic Stress, 5(3), 377-391.

18. Larraín, S. (1992). Informe preliminar de investigación en violencia intrafamiliar y la situación de la mujer en Chile. Santiago de Chile: SERNAM.

19. Levendosky, A., Lynch, S. \& Graham-Bermann, S. (2000). Mothers' Perceptions of the impact of Woman Abuse on Their Parenting. Violence Against Women, 6(3), $247-271$.

20. Lhor, B.C. (2001). A comparison of neuropsychological functioning of high and low risk mothers for child physical abuse. Dissertation Abstracts International: Section B: the Sciences \& Engineering, 62 (5-B), 2.490 .

21. Matud, M. (2004). Impacto de la violencia doméstica en la salud de la mujer maltratada. Psicothema, 16(3), 397-401.

22. Matud, M., Belén, A. \& Padilla, V. (2004). Intervención psicológica con mujeres maltratadas por su pareja Papeles del psicólogo, 25(88),1-9.

23. Merino, C., Díaz, M. \& DeRoma, V. (2004). Validación del Inventario de Conductas Parentales: Un análisis factorial confirmatorio. Personas, 7, 145-164.

24. Milner, J.S. (1993). Social information processing and physical child abuse. Clinical Psychology Review, 13(3), 275-294.

25. Musito, G., Martínez, B. \& Murgui, S. (2006). Conflicto marital, apoyo parental y ajuste escolar en adolescentes. Anuario de Psicología de la Universidad de Barcelona, 36(3), $247-258$.

26. Nayak, M.B. \& Milner, J.S. (1998). Neuropsychologycal functioning: comparison of mothers at high and lowrisk for child physical abuse. Child Abuse and Neglect, 22(7), 687-703.

27. Patró, R., Corbalán, F. \& Limiñana, R. (2005). Víctimas de violencia familiar: Consecuencias psicológicas en hijos de mujeres maltratadas. Anales de Psicología; $21,1,11-17$.

28. Patró, R., Corbalán, F. \& Limiñana, R. (2007). Depresión en mujeres maltratadas. Relaciones con estilos de personalidad, variables contextuales y de la situación de violencia. Anales de Psicología; 23,1, $118-124$.

29. Pham, K.T. (2000). The relationship among child abuse potential, marital satisfaction and domestic violence: using child abuse potential to predict domestic violence. Dissertation Abstracts International: Section B: the Sciences \& Engineering, 61 (3-A), 898.

30. Pons-Salvador, G., Cerezo, A. \& Bernabé, G. (2005). Cambio y estabilidad en los factores que afectan negativamente a la parentalidad. Psicothema, $17,1,31-36$.

31. Roca, M. (2002). Autoeficacia: su valor para la eficacia cognitiva conductual. Revista Cubana de Psicología,19(3).

32. Romero, I. (2004). Desvelar la violencia: Una intervención para la prevención y el cambio. Papeles del Psicólogo, 88. (Citado en Marzo del 2009) Disponible en: http:/www.papelesdelpsicologo.es/ vernumero.asp? $\mathrm{id}=1156$

33. Sarasua, B., Zubizarreta, I., Echeburúa, E. \& Corral, P. (2007). Perfil psicopatológico diferencial de las víctimas de violencia de pareja en función de la edad. Psicothema; 19(9), 459 - 466.

34. Sprinkle, J. (2007). Domestic Violence, Gun Ownership, and Parental Educational Attainment: How do They Affect the Aggressive Beliefs and Behaviors of Children? Child and Adolescent Social Work Journal; 24(2), $133-151$.

35. Valor - Segura, I., Expósito, F. \& Moya, M. (2008). Attribution of aggressor's behavior and advice to the victim in a case of domestic violence. Revista de Psicología Social, 2008, 23(2), 171-180.

36. Zarza, M. \& Froján, M. (2005). Estudio de la violencia doméstica en una muestra de mujeres latinas residentes en Estados Unidos. Anales de Psicología, $21,1,18-26$. 